\title{
An Enhanced Factor Analysis of Performance Degradation Assessment on Slurry Pump Impellers
}

\author{
Shilong Sun, Peter W. Tse, and Y. L. Tse \\ Smart Engineering Asset Management Laboratory (SEAM), Department of Systems Engineering \& Engineering Management, \\ City University of Hong Kong, Kowloon Tong, Hong Kong \\ Correspondence should be addressed to Peter W. Tse; meptse@cityu.edu.hk
}

Received 25 July 2016; Accepted 13 December 2016; Published 4 January 2017

Academic Editor: Mickaël Lallart

Copyright (C) 2017 Shilong Sun et al. This is an open access article distributed under the Creative Commons Attribution License, which permits unrestricted use, distribution, and reproduction in any medium, provided the original work is properly cited.

Slurry pumps, such as oil sand pumps, are widely used in industry to convert electrical energy to slurry potential and kinetic energy. Because of adverse working conditions, slurry pump impellers are prone to suffer wear, which may result in slurry pump breakdowns. To prevent any unexpected breakdowns, slurry pump impeller performance degradation assessment should be immediately conducted to monitor the current health condition and to ensure the safety and reliability of slurry pumps. In this paper, to provide an alternative to the impeller health indicator, an enhanced factor analysis based impeller indicator (EFABII) is proposed. Firstly, a low-pass filter is employed to improve the signal to noise ratios of slurry pump vibration signals. Secondly, redundant statistical features are extracted from the filtered vibration signals. To reduce the redundancy of the statistic features, the enhanced factor analysis is performed to generate new statistical features. Moreover, the statistic features can be automatically grouped and developed a new indicator called EFABII. Data collected from industrial oil sand pumps are used to validate the effectiveness of the proposed method. The results show that the proposed method is able to track the current health condition of slurry pump impellers.

\section{Introduction}

Slurry pumps, such as oil sand pumps, are widely used in industry to convert electrical energy to slurry potential and kinetic energy. Slurry pumps work under adverse conditions, so their impellers are prone to suffer wear, resulting in breakdown. Therefore, fault diagnosis and prognosis of slurry pump impellers are major concerns. Recent research has classified different slurry pump impeller health conditions. $\mathrm{Qu}$ and Zuo [1] proposed a data cleaning algorithm, based on support vector machines and random subsampling validation, to classify and identify different impeller health conditions. A modified neighborhood rough set model was proposed by Zhao et al. [2] to select useful features for the identification of different impeller health conditions. They found that the modified model can produce higher prediction accuracies than the original neighborhood rough set model. In addition to fault diagnoses of slurry pump impellers, prognostic studies of slurry pumps have also been carried out. These prognostic works can be broadly divided into two steps
[3]. The first is to assess performance degradation, and the second is to estimate the remaining useful life of the pumps. In this study, only the performance degradation assessment of slurry pump impellers is investigated.

Performance degradation assessment tracks the health conditions of components and systems. A number of typical examples show how different methods have been developed for performance degradation assessment. Zhao et al. [4] used half and full spectra, fuzzy preference based on rough sets, and principle component analysis (PCA) to develop a new indicator for impeller damage to pumps. The results illustrate that the indicator is capable of monitoring the health status of pump impellers monotonically. Miao et al. [5] used hidden Markov Models to propose a novel probability health description index to monitor gear health conditions. Based on the discrete wavelet transform, Wang et al. [6] developed a health indictor known as the frequency spectrum growth index (FSGI) for the evaluation of gear health conditions. Wang et al. [7] went on to fuse different statistical features, describing the gear health conditions using support vector 
data descriptions. Miao et al. [8] combined comblet filtering and an exponentially weighted moving average method, to develop a health conditions indicator (HCI) for bearing degradation assessment. Lei et al. [9] proposed two diagnostic parameters to track the health conditions of planetary gearboxes, in terms of the statistical characteristics of planetary gearbox vibration signals in the domains of both time and frequency. Based on the industrial run to alert slurry pump data, Tse and Wang $[3,10]$ also designed a specific moving average wear degradation index and an impeller health indicator, to assess slurry pump impeller degradation. The impeller health indicator is constructed by using the PCA of the statistical features extracted from the slurry pump vibration signals. Therefore, it is vital that performance degradation assessment extracts and develops an effective health condition indicator from raw signals.

The slurry pump original vibration signals are part of a high dimensional data set. Moreover, the common statistical indicators for monitoring the degradation were not effective in tracking the health conditions of the slurry pumps. To minimize the computational burden and to discover an effective indicator for monitoring the degradation of the slurry pump, methods such as feature clustering are used. Feature clustering, such as PCA and independent component analysis, are widely used to investigate the correlation of selected attributes and to reduce the dimension of the feature space. For example, Tse and Wang [11] applied the PCA method to construct a health indicator to monitor the health evolution of an impeller. Chang et al. [12] used the PCA combined with a support vector machine to extract the first principal feature associated with the operating mine system gearbox. The PCA algorithm was found to be effective in removing redundancy and reducing the dimensionalities of the feature space. Harmouche et al. $[13,14]$ proposed a fault detection approach using the PCA, based on probability distribution, which can successfully detect incipient faults that are undetectable by traditional methods. Lee et al. [15] used a modified independent component analysis algorithm to extract important independent components from a multivariate statistical data set, which was applied to fault diagnosis in a wastewater treatment process. However, the abovementioned methods cannot ensure that the representation of the whole content of the original variable data is preserved and are not able to cluster features into a single new effective indicator when assessing slurry pump impeller degradation. Therefore, the traditional statistical method of factor analysis (FA), based on the indicators extracted from the slurry pump vibration data, is proposed in this paper. FA [16] is a widely used statistical tool in different fields, such as economics [17], business [18], psychology [19], social sciences [20, 21], and medicine $[22,23]$. However, it is rarely applied to fault diagnosis and prognosis of complex mechanics system, particularly feature extraction and selection. Apley and Shi [24] proposed a creative FA, which can extract diagnostic features from a large volume of automated in-process measurement data. Zhang et al. [25] studied a novel fault FA scheme, which can reduce the influence on fault detection of the relay protection's wrong action device. Kuravsky et al. [26] proposed a wavelet-based confirmatory FA for monitoring the damage accumulation factors responsible for the evolution of technical and other systems. In sum, the FA has been proven effective in combining potential latent variables, covering the whole content of the original variables, similar to the PCA. Compared to the PCA, which focuses on the ranking and selection of the components, the FA could classify, group, and remove the redundancy of the data information. The calculation process of the FA is more complicated than the PCA method, with an extra step, known as factor rotation. This kind of grouping function could increase the efficiency of the new indicator, developed through FA, in the evaluation of the degradation of the slurry pump impellers. In addition, the FA measures underlying factors, in which the identification of such underlying factors simplifies the understanding and description of the complex original variables and also reduces the number of factors. In other words, the FA algorithm can be taken as a data-reduction technique, as it reduces a large number of correlated variables to a smaller set of factors that reflect the original variables. In this study, combining the FA with a low-pass moving average filter is proposed and used to track the health conditions of a slurry pump, with less dimensions and a more effective new health indicator.

A low-pass filter is applied to the slurry pump vibration signals. To provide an alternative to the impeller health indicator, an enhanced factor analysis based impeller indicator (EFABII) is proposed in this study. The procedures of the proposed method are similar to those reported in our previous publication. The major difference is that the FA can be used to reduce the dimensionality of the statistical features. In addition, it can also be used to categorize the statistical features. Therefore, the relationship between different statistical features can be clearly identified.

The rest of the paper is organized as follows. In Section 2, the FA is reviewed and discussed. A method used for slurry pump impeller performance degradation assessment is proposed in Section 3. In Section 4, industrial data are used to validate the effectiveness of the proposed method. Conclusions are drawn in Section 5.

\section{Factor Analysis Principle}

Factor analysis $[27,28]$ is a method that uses a lower number of new variables, known as common factors, to describe the variability of the observed and correlated variables. The observed variables are therefore the linear combinations of the new factors and errors. The FA is generally divided into four steps: (1) calculation of correlation matrix for all variables; (2) factor extraction; (3) factor rotation; (4) establishment of latent factors. Based on the FA principles, an enhanced factor analysis method was proposed in this paper.

Suppose that $X_{i}(i=1,2, \ldots, p)$ are $p$ observed and correlated variables. The FA aims to solve the following mathematical problem. The matrix form of the FA equation is derived as follows [27]: 


$$
\begin{aligned}
\mathbf{X}-\boldsymbol{\mu}= & \left(\begin{array}{c}
X_{1} \\
X_{2} \\
\vdots \\
X_{p}
\end{array}\right)-\left(\begin{array}{c}
\mu_{1} \\
\mu_{2} \\
\vdots \\
\mu_{p}
\end{array}\right) \\
= & \left(\begin{array}{cccc}
a_{11} & a_{12} & \cdots & a_{1 m} \\
a_{21} & a_{22} & \cdots & a_{2 m} \\
\vdots & \vdots & & \vdots \\
a_{p 1} & a_{p 2} & \cdots & a_{p m}
\end{array}\right)\left(\begin{array}{c}
F_{1} \\
F_{2} \\
\vdots \\
F_{m}
\end{array}\right) \\
& +\left(\begin{array}{c}
\varepsilon_{1} \\
\varepsilon_{2} \\
\vdots \\
\varepsilon_{p}
\end{array}\right)=\mathbf{A F}+\boldsymbol{\varepsilon},
\end{aligned}
$$

where $\mathbf{X}$ is the vector of the observed variables; $\boldsymbol{\mu}$ is the mean vector of the observed variables; $\mathbf{A}$ is the factor loadings of a constant $p$-by- $m$ matrix; $\mathbf{F}$ is a vector of independent and standardized common factors which is the unobservable latent variable; $\boldsymbol{\varepsilon}$ is a vector of specific factors which cannot be included by those common factors; $m$ is the number of the original features and is equal to 18 in this paper, and $p$ is the observed variables.

Here, the vectors $\mathbf{F}$ and $\boldsymbol{\varepsilon}$ must satisfy the following requirements [27]:

$$
\begin{aligned}
& \operatorname{cov}\left(F_{j}, F_{k}\right)=0, \quad j \neq k ; E(\mathbf{F})=0, D(\mathbf{F})=1, \\
& \operatorname{cov}\left(\varepsilon_{j}, \varepsilon_{k}\right)=0, \quad j \neq k ; E(\boldsymbol{\varepsilon})=0, D(\boldsymbol{\varepsilon})=\sigma^{2} .
\end{aligned}
$$

In addition, because the common factor and specific factor are irrelevant, then

$$
\operatorname{cov}(\mathbf{F}, \boldsymbol{\varepsilon})=0 .
$$

Common factor $\mathbf{F}$ accounts for the correlation among the variables. The larger the coefficient $a_{i j}$ of each common factor $F_{i}$, the more explained the content between that common factor and that corresponding variable. The specific factor $\boldsymbol{\varepsilon}$ explains the remaining variance and the error of that variable. As aforementioned, the factor loading is here referred to as the correlation. Factor loading $a_{i j}$ is the correlation coefficient between the $i$ th variables and the $j$ th common factor. It reflects the relative importance of the $i$ th variables and the $j$ th common factor. The closer to 1 its absolute value is, the higher relative degree it has. The factor loadings equation can be obtained from

$$
\mathbf{A}=(\mathbf{X}-\boldsymbol{\mu}-\boldsymbol{\varepsilon}) \cdot \mathbf{F}^{-\mathbf{1}}
$$

From (2), the variance of $\mathbf{X}$ can be deduced, which is as follows [28]:

$$
\begin{aligned}
D\left(\mathbf{X}_{i}\right)=\left(a_{i 1}^{2}+a_{i 2}^{2}+\cdots+a_{i m}^{2}\right)+\sigma_{i}^{2}= & h_{i}^{2}+\sigma_{i}^{2}, \\
& (i=1,2, \ldots, p) .
\end{aligned}
$$

Then, the total variance of $\mathbf{X}$ is

$$
D(\mathbf{X})=\text { Communality }+ \text { Specific variance. }
$$

Therefore, the commonality is the sum square of the coefficients or factor loadings of the common factors. The common variance $h_{i}^{2}$ reflects the explained extent of the common factor to the total variance of the variables. The larger $h_{i}^{2}$ is, the higher the correlation between that kind of factor and its variables is.

The establishment of the enhanced FA model identifies the common factors and classifies the variables but more importantly enables the meaning of every common factor to be studied, and further analysis can be carried out. The original variables are considered as a linear combination of common factors through this model. To acquire a better result through the enhanced FA model, the factor-loading matrix should be rotated. The common factors can then explain the original variables specifically and precisely. The methods of factor rotation can be divided into orthogonal and oblique. Therefore, to simplify the rotation of the factorloading matrix, the polarized squared value of each column and row of the factor-loading matrix should be set up close to 0 or $1[16,27-29]$. For the method described in this study, "Equimax" is chosen as the rotation method, as this is a compromise between polarizing the rows' and columns' factor matrix.

The last step of the enhanced FA model is to acquire the factor scores. These can be used to conduct further studies, such as regression or the evaluation of sample classification analysis.

According to (1) and (4), the factor scores function can be written as follows:

$$
\mathbf{F}=\mathbf{A}^{-1} \cdot(\mathbf{X}-\boldsymbol{\mu}-\boldsymbol{\varepsilon}) .
$$

The coefficients of the factors should be first deduced so each of the factor scores can then be obtained.

\section{The Proposed Method for Impeller Performance Degradation Assessment}

In this study, a new statistics impeller health indicator is developed for describing the degradation performance assessment of slurry pumps. This new health indicator is built according to our previous studies $[3,7,10]$. The vibration measurement data were collected from the application of the smart asset management system (SAMS) software developed by the Smart Engineering Asset Management Lab. The data acquisition instruments included a National Instrument (NI) DAQ 9172 and a DAQ module NI 9234. Four accelerometers were installed in four different positions on the slurry pumps, to monitor the various working conditions. The data measurements were recorded using a number series from 1 to 1101 to identify their source. The recording period was three months and the sampling rate was $51,200 \mathrm{~Hz}$. Vibration data solely from channel 3 were used in this study. The details of the steps for processing the data are as follows.

Suppose $Y_{k}(n)$ is the original vibration data of the slurry pump, and let $P$ denote the total number of measurement files 
and $k=1,2,3, \ldots, P$, and define $n=1,2, \ldots, N . N$ is equal to 51,200 , which is the sampling rate of the measurement files.

A low-pass filter with a cut-off frequency of $110 \mathrm{~Hz}$ was applied to process the slurry pump original vibration data $Y_{k}(n)$. Nine statistical features in the time domain and their corresponding frequencies were then extracted from the processed vibration signals $X_{n}$. Normalization was conducted before applying the FA.

The original feature matrices in time and frequency domains are, respectively, constructed as

$$
\begin{aligned}
F_{\mathrm{LP}}(t) & =[\mu, \sigma, \mathrm{RMS}, \mathrm{SK}, \mathrm{KU}, \mathrm{CF}, \mathrm{CLF}, \mathrm{SF}, \mathrm{IF}], \\
F_{\mathrm{LP}}(f) & =[\mu, \sigma, \mathrm{RMS}, \mathrm{SK}, \mathrm{KU}, \mathrm{CF}, \mathrm{CLF}, \mathrm{SF}, \mathrm{IF}],
\end{aligned}
$$

where $F_{\mathrm{LP}}(t)$ represents the features in time domain and $F_{\mathrm{LP}}(f)$ represents the features in frequency domain. The subscript LP represents low-pass filtering. The following equations for the nine statistical features were extracted from signals in time and frequency domains after the low-pass filter was initially applied to process raw vibration data $\left(X_{n}\right.$ represents the processed vibration data in temporal and spectral domains).

(1) Mean:

$$
\mu=\frac{1}{N} \sum_{n=1}^{N} X_{n}
$$

(2) Standard deviation (STD):

$$
\sigma=\sqrt{\frac{1}{N-1} \sum_{n=1}^{N}\left(X_{n}-\mu\right)^{2}} .
$$

(3) Root mean square:

$$
\mathrm{RMS}=\sqrt{\frac{1}{N} \sum_{n=1}^{N}\left(X_{n}\right)^{2} .}
$$

(4) Skewness:

$$
\mathrm{SK}=\frac{\sum_{n=1}^{N}\left(X_{n}-\mu\right)^{3}}{(N-1) \sigma^{3}} .
$$

(5) Kurtosis:

$$
\mathrm{KU}=\frac{\sum_{n=1}^{N}\left(X_{n}-\mu\right)^{4}}{(N-1) \sigma^{4}} .
$$

(6) Crest factor:

$$
\mathrm{CF}=\frac{\max \left(\left|X_{n}\right|\right)}{\sqrt{(1 / N) \sum_{n=1}^{N}\left(X_{n}\right)^{2}}} .
$$

(7) Clearance factor:

$$
\operatorname{CLF}=\frac{\max \left(\left|X_{n}\right|\right)}{\left((1 / N) \sum_{n=1}^{N} \sqrt{\left|X_{n}\right|}\right)^{2}} .
$$

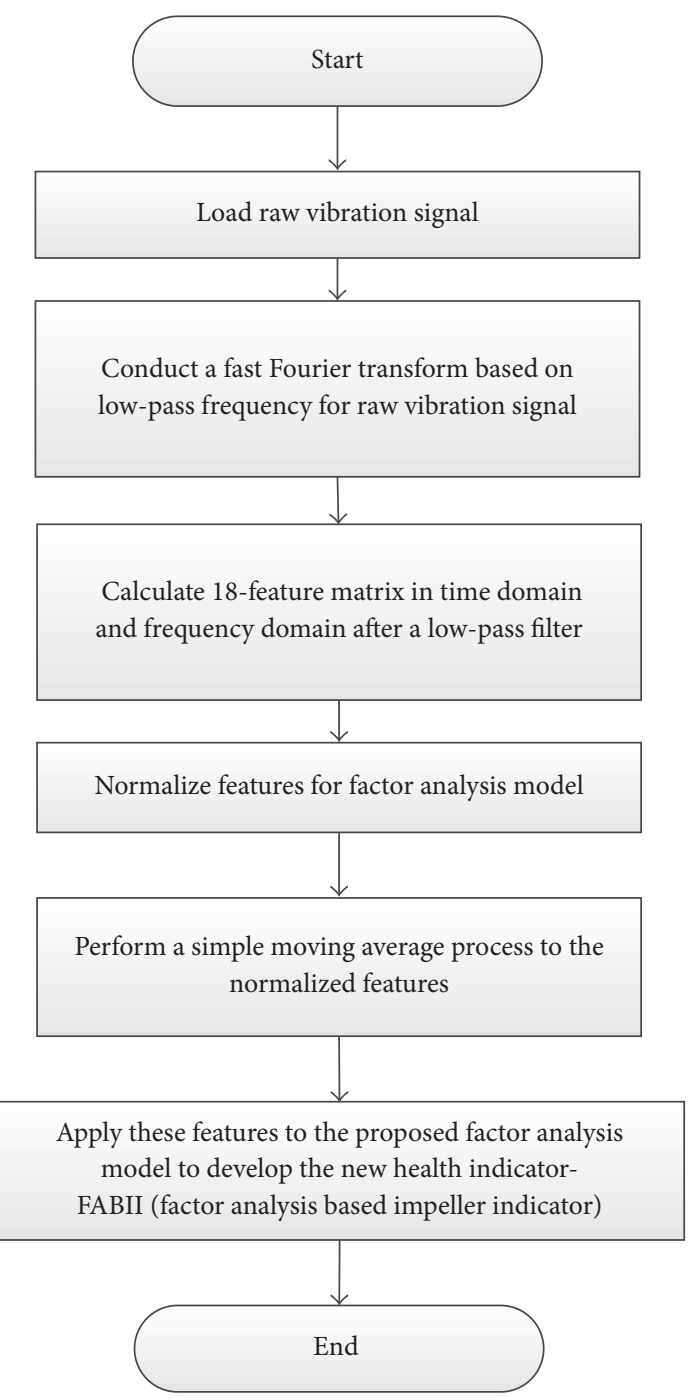

Figure 1: Procedures of the fault diagnosis on a slurry pump with the enhanced FA model.

(8) Shape factor:

$$
\mathrm{SF}=\frac{\sqrt{(1 / N) \sum_{n=1}^{N}\left(X_{n}\right)^{2}}}{(1 / N) \sum_{n=1}^{N}\left|X_{n}\right|} .
$$

(9) Impulse factor:

$$
\mathrm{IF}=\frac{\max \left(\left|X_{n}\right|\right)}{(1 / N) \sum_{n=1}^{N}\left|X_{n}\right|} .
$$

These features are used to construct a new feature matrix:

$$
X_{k}=\left[F_{\mathrm{LP} k}(t), F_{\mathrm{LP} k}(f)\right], \quad k=1,2,3, \ldots, P .
$$




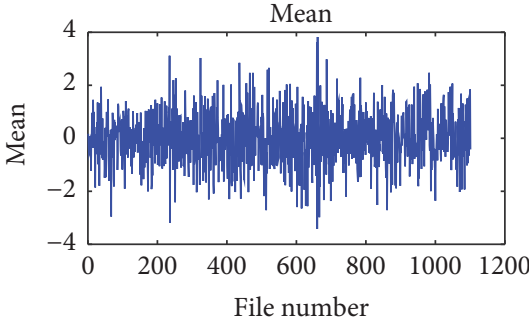

(a)

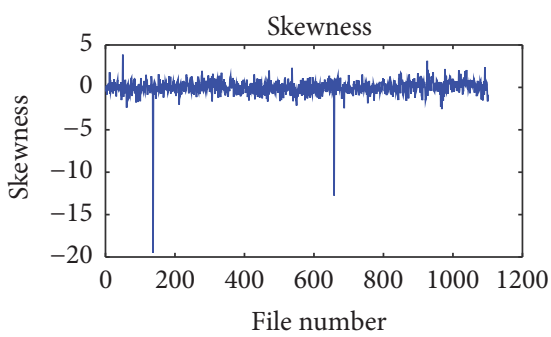

(d)

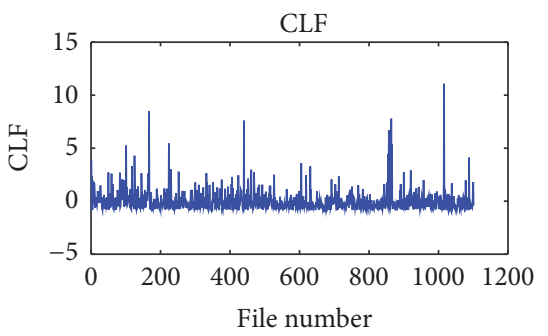

(g)

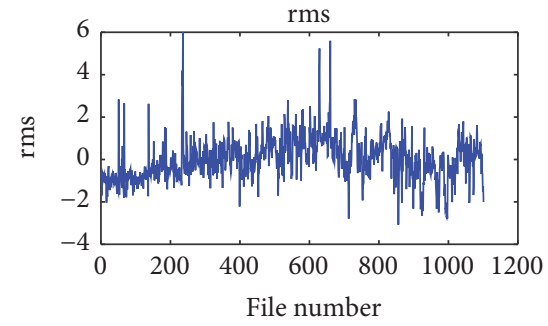

(b)

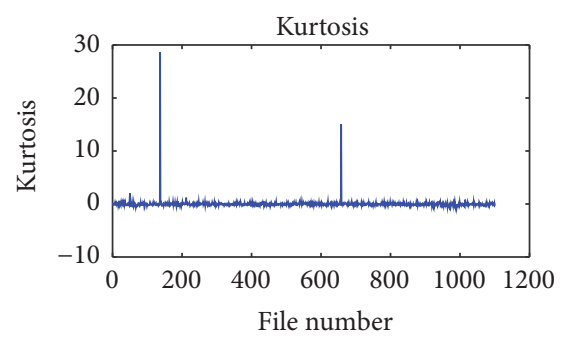

(e)

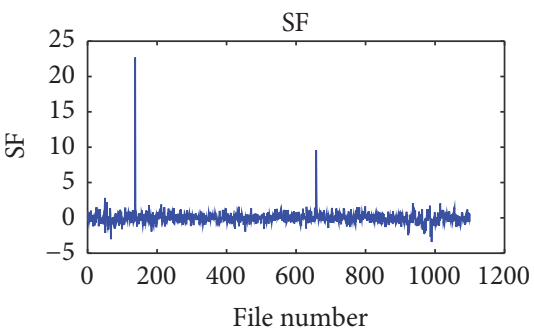

(h)

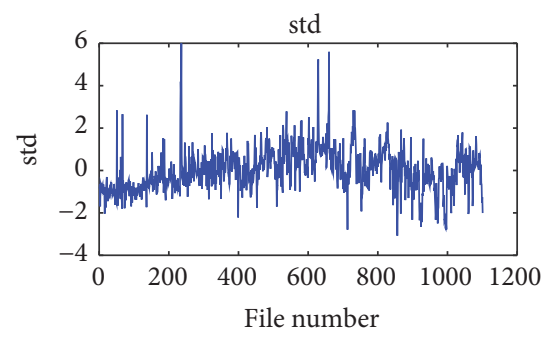

(c)

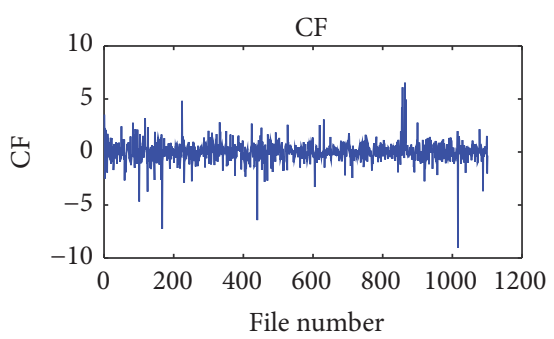

(f)

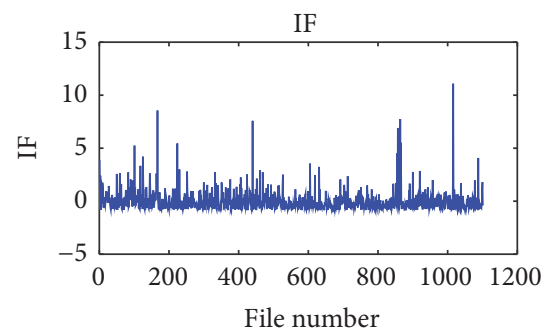

(i)

Figure 2: The nine statistical features extracted from the low-pass filter processed data versus the successive file numbers in the time domain (mean, rms, std, skewness, kurtosis, CF, CLF, SF, and IF from (a)-(i)).

The new feature matrix is then normalized according to

$$
\begin{gathered}
X_{k, j}=\frac{\left(X_{k, j}-\sum_{k=1}^{P} X_{k, j} / P\right)}{\sqrt{\sum_{t=1}^{P}\left(X_{k, j}-\sum_{k=1}^{P} X_{k, j} / P\right)^{2} / P}} \\
\quad k=1,2,3, \ldots, P, \quad j=1,2,3, \ldots, 18 .
\end{gathered}
$$

A moving average process is used for each column of the new matrix from (20):

$$
\begin{aligned}
& X M A_{k, j}=\frac{\sum_{1}^{k} X_{k, j}}{k} \\
& \quad k=1,2,3, \ldots, P ; j=1,2,3, \ldots, 18 .
\end{aligned}
$$

Through (21), a new matrix $X M A_{k, j}$ is generated for the study of the EFA based impeller indicator. Then the enhanced factor analysis based impeller indicator (EFABII) can be calculated by combining the result from (21) and (7). The details of the calculation procedures are summarized in Figure 1.

\section{Applying the Proposed Method on Industrial Data}

The performance degradation assessment is vital for monitoring the health conditions of slurry pumps, but it is very difficult to find or extract an effective indicator to describe the propagation performance throughout the whole life of a slurry pump [30-32]. The extraction of the enhanced factor analysis based impeller indicator (EFABII) was briefly introduced in Sections 2 and 3. To validate the effectiveness of this EFABII, a case study was carried out. The results obtained by the EFABII demonstrated an obvious damage growth and propagation performance trend and are explained in this section.

4.1. EFABII Applied in the Time Domain. The proposed method was applied to analyze the data described in Section 3. The features obtained from the raw data, and their respective normalized moving average indicators after being processed by the low-pass filter, are shown in Figures 2 and 3.

Figure 2 illustrates that the trends of the performance wear degradation were not obvious. A normalization step was used to eliminate the effect of units for the variables. A simple moving average process was then used for these nine feature trends, and the results are shown in Figure 3. The 


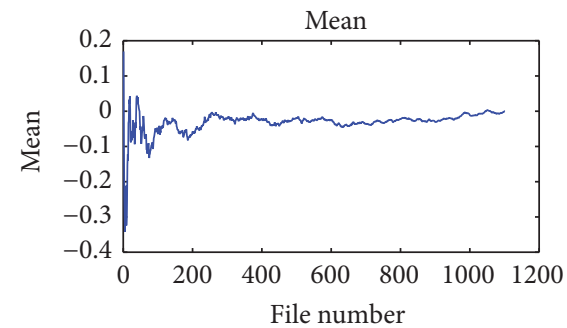

(a)

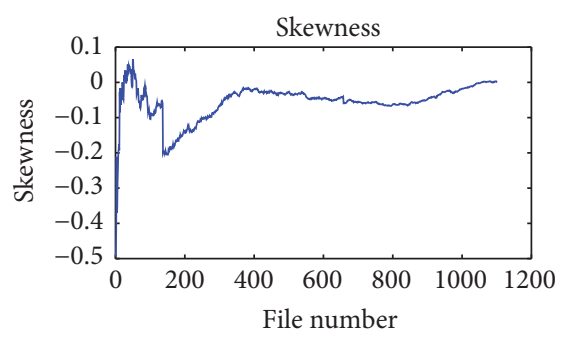

(d)

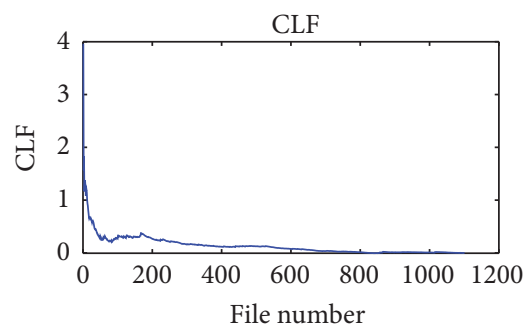

(g)

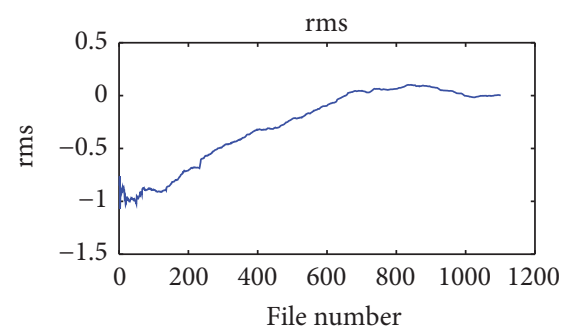

(b)

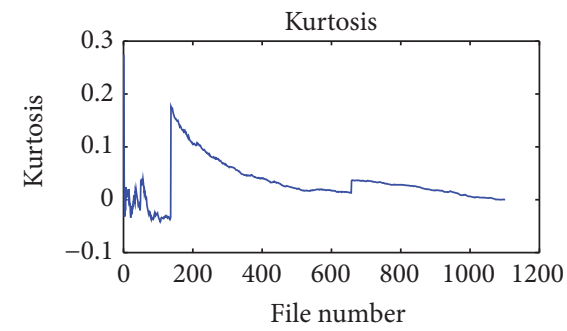

(e)

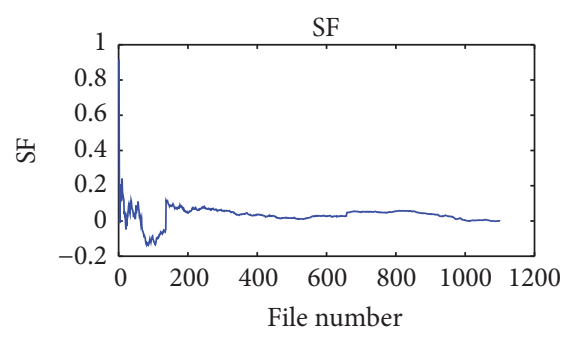

(h)

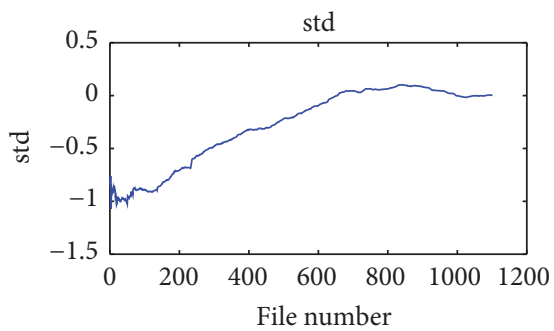

(c)

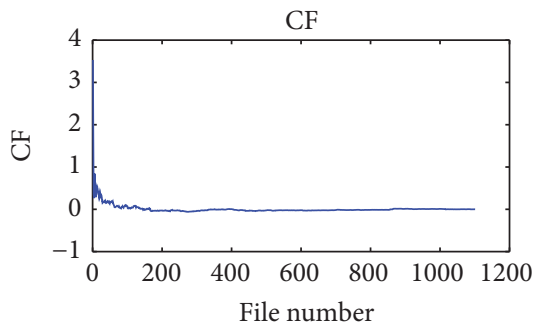

(f)

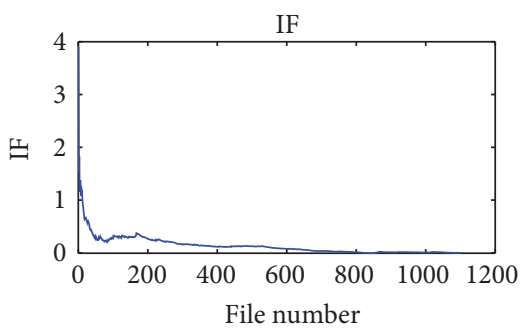

(i)

FIGURE 3: The nine statistical feature indicators from Figure 2 after normalization, processed with the moving average process (mean, rms, std, skewness, kurtosis, CF, CLF, SF, and IF from (a)-(i)).

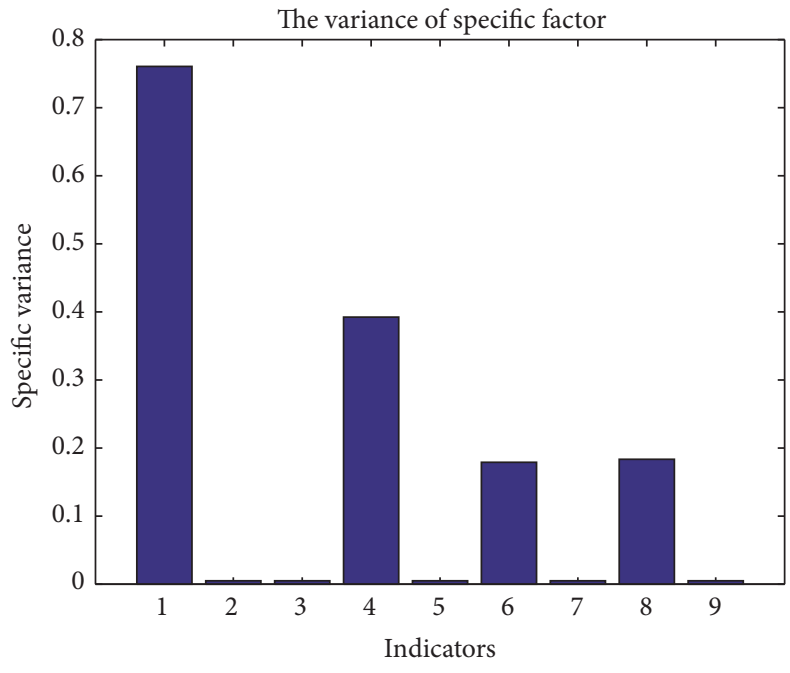

(a)

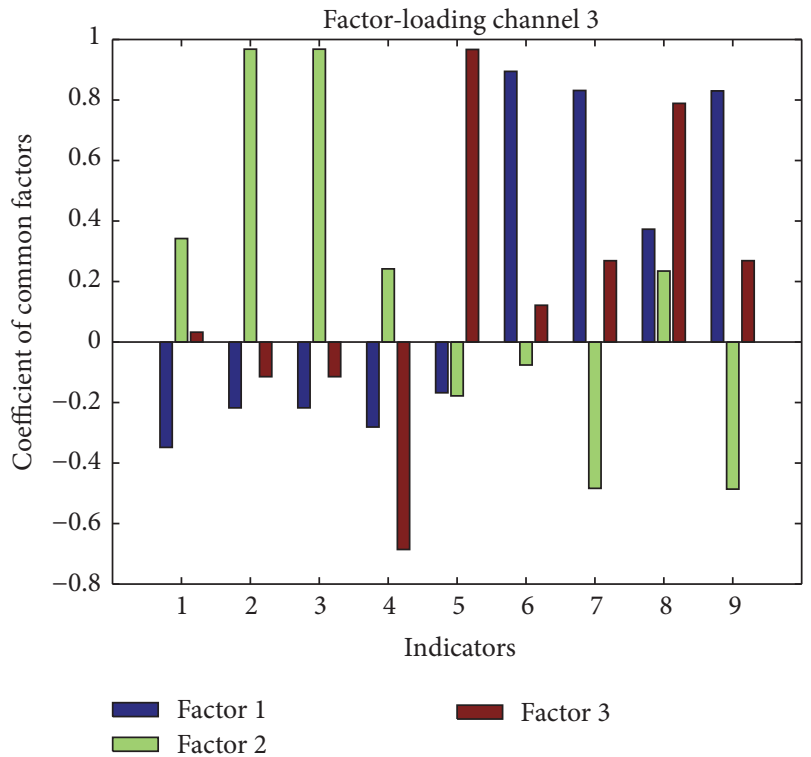

(b)

Figure 4: (a) The specific variances of the indicators. (b) The coefficients of the common factors. 


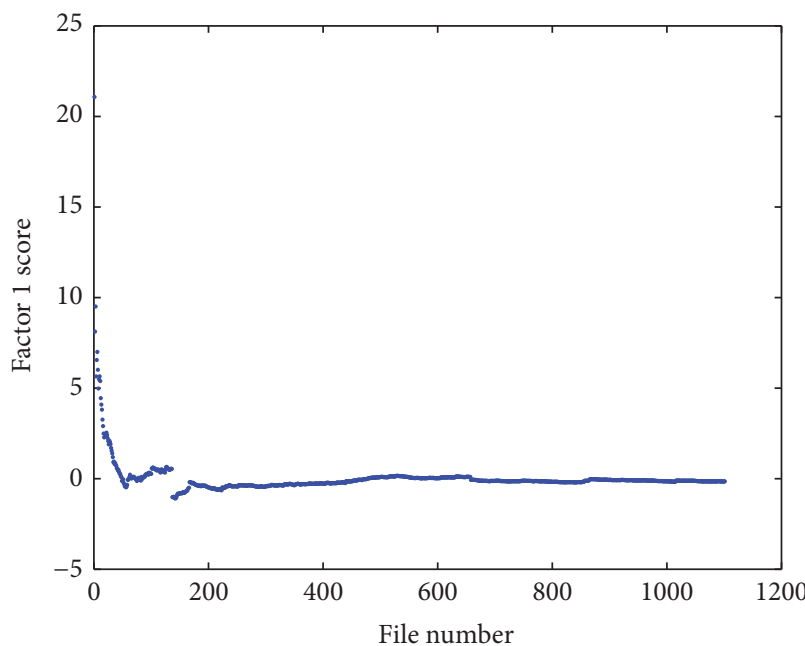

- Factor 1

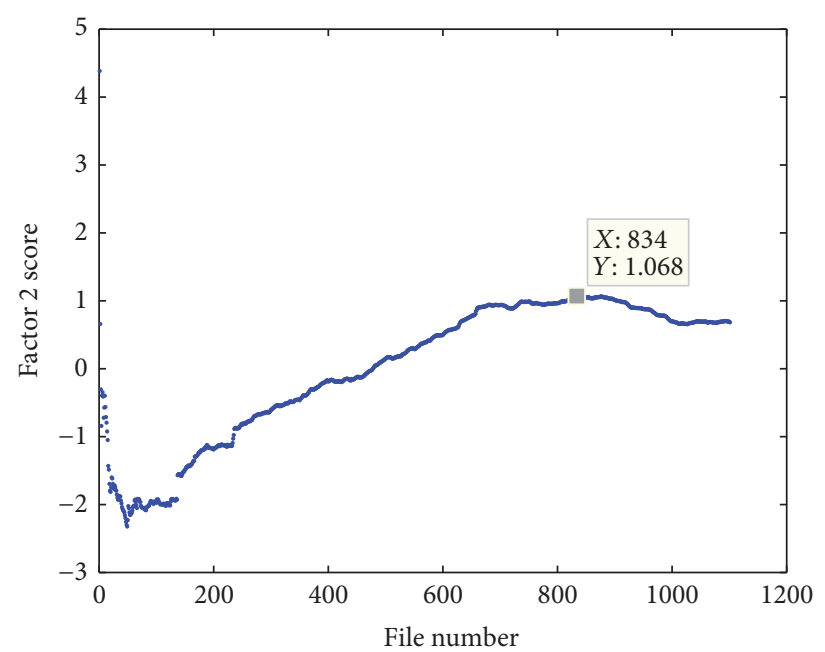

- Factor 2

(a)

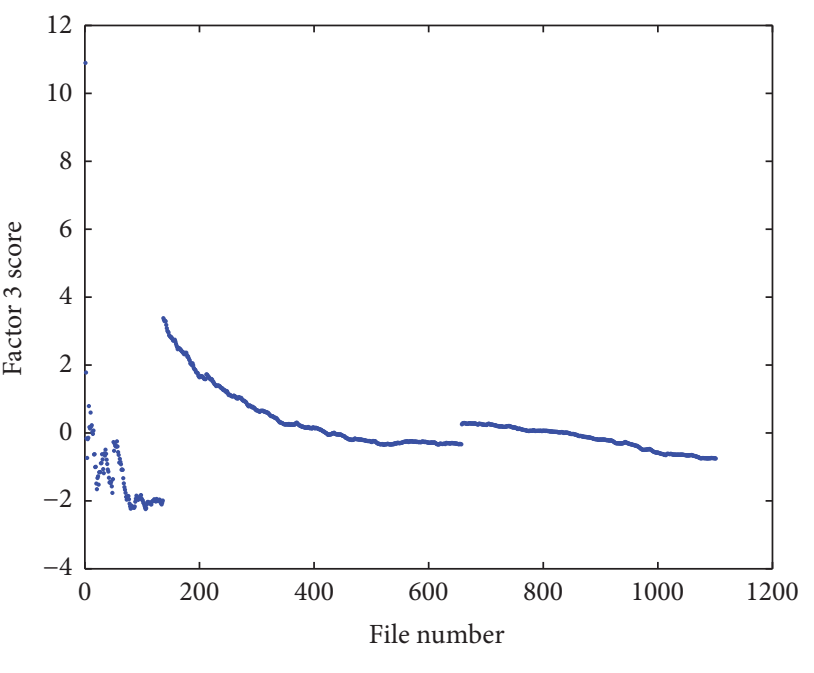

- Factor 3

(c)

FIGURE 5: The results of the EFABII in time domain for (a) factor 1, (b) factor 2, and (c) factor 3, respectively.

trends for the features rms and std shown in Figure 3 were found to be very similar and exhibited obvious and clear wear degradation performance throughout the whole operating stage of slurry pumps. This is because they have a similar formula. If the mean of a signal equals 0 , rms and std are the same with each other.

The results obtained by using the EFA are shown in Figures 4(a) and 4(b). The specific variances of the nine features in the time domain are shown in Figure 4(a). As mentioned, if the specific variance value is close to " 1 " there is no common factor in that specific kind of variable, while a " 0 " value means that the features could be entirely determined by the common factor. The coefficients of the common factor of the nine features in the time domain are shown in Figure 4(b). The closer to " 1 " the coefficients absolute values are, the more the correlation between that factor and the original features. Figure 4(b) clearly shows that the features 6, 7, and 9 (CF, CLF, and IF) can be classified as factor 1, while factor 2 includes the features 2 and 3 (rms and std). Factor 3 has a good correlation with the features 4,5 , and 8 (skewness, kurtosis, and SF). Indicator 1 in Figure 4(a) has a high specific variance (lager than 0.75), and its coefficient of common factor is very small (less than 0.5), as shown in Figure 4(b), so it cannot be classified by any factor and must be eliminated.

Factor 2 in Figure 5(b) clearly has a similar trend to the features rms and std described in Figure 3, so it can replace the original features 2 and 3 (rms and std). The value of factor 2 increases with the file number, and reaches its maximum value of 1.068 at the 834 th file. The other two factors do not demonstrate similar monotonic damage growth trends. Therefore, factor 2 can be applied to monitor the slurry pumps' working conditions. This factor can help to reduce the multivariate data dimensions and the nine statistical features need not be used. 


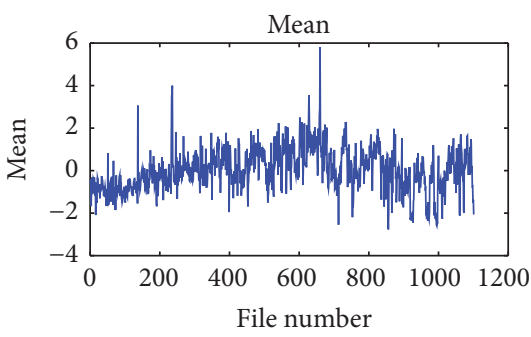

(a)

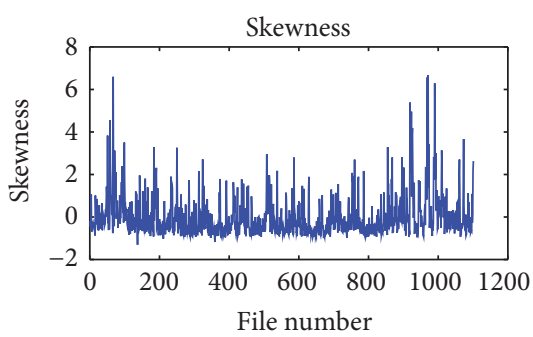

(d)

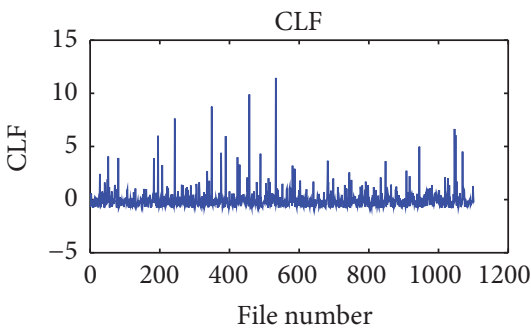

(g)

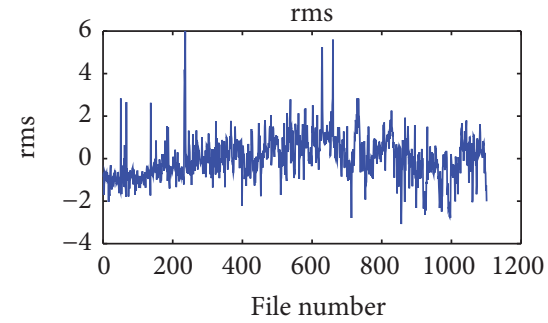

(b)

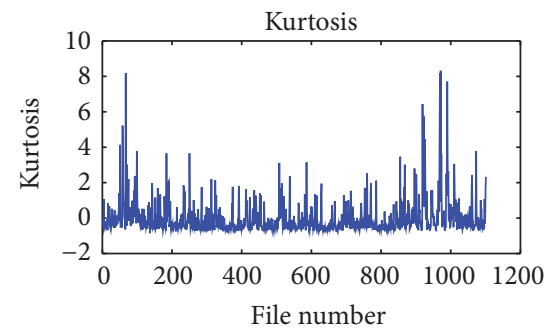

(e)

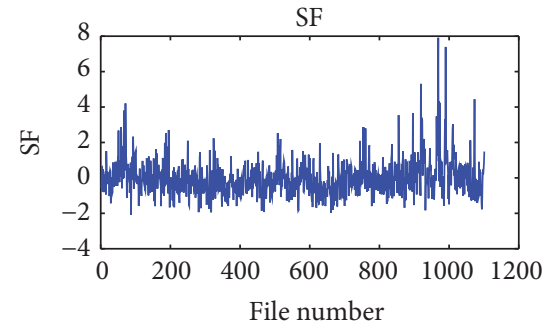

(h)

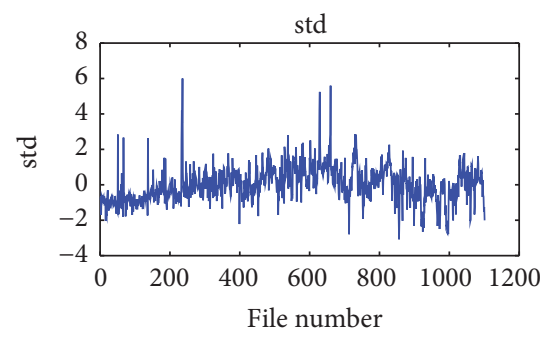

(c)

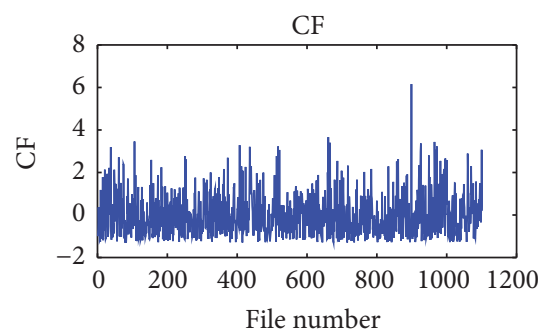

(f)

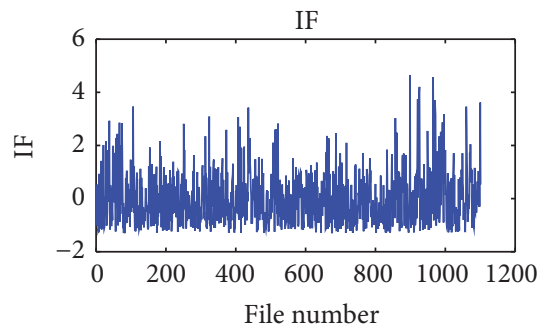

(i)

FIGURE 6: The nine statistical features extracted from the low-pass filter processed data versus the successive file numbers in the frequency domain (mean, rms, std, skewness, kurtosis, CF, CLF, SF, and IF from (a)-(i)).

In conclusion, factor 2 in the time domain is selected as the new EFABII for monitoring the health conditions of pumps throughout the operation stage.

4.2. EFABII Applied in the Frequency Domain. The features obtained from the raw data, and their normalized moving average indicators in the frequency domain after being processed by the low-pass filter, are shown in Figures 6 and 7 , respectively. As described in the previous section, a simple moving average was applied as the trend for evaluating the performance wear degradation was not obvious.

The results are shown in Figure 7 and were obtained after the features extracted from the frequency spectra are processed with the moving average method. Monotonic trends are found from the features mean, rms, and std in this figure. In Figure 7, rms and std should have the above same explanation. Besides, the mean of the frequency domain also exhibits the similar trend, which is different from the mean extracted from the time domain. This is because the frequency of zero in the frequency domain is equal to the mean of the signal in the time domain according to the fast Fourier transform. So, the mean of the frequency domain takes all frequencies into consideration rather than only the frequency of zero. Consequently, the mean of the signal in the time domain is different from that in the frequency domain. Obvious wear degradation performance throughout the operating stage of a slurry pump can be detected using these three features. The three trends are also similar, and demonstrate a rising initial trend, which becomes saturated after reaching the maximum point at around the 850th file, and then slightly decreases.

The results obtained by using the EFA are shown in Figures $8(\mathrm{a})$ and $8(\mathrm{~b})$. The features mean, rms, and std (1, 2, and 3) can be grouped by factor 1 . The skewness, kurtosis, and $\operatorname{SF}(4,5$, and 8$)$ can be grouped by factor 2 , with the remaining features CF, CLF, and IF (6, 7, and 9) grouped by factor 3. Monotonic trends are absent from factors 2 and 3, so these two factors cannot be used to fully represent the content of the slurry pump degradation wear assessment. The trends obtained by factors 1,2 , and 3 after using the EFA are shown in Figure 9. Factor 1 clearly possesses similar trends to the features mean, rms, and std, unlike the other two factors in Figure 7. The maximum value of factor 1 is found to be 1.043 , located at the 834 th file. This particular file number is the same as for factor 2 in the time domain, as reported in Section 4.1. Factor 1 in the frequency domain can therefore entirely reflect the trend of the features mean, rms, and std in this particular low frequency zone. Therefore factor 1 in the frequency domain is selected as the most effective new 


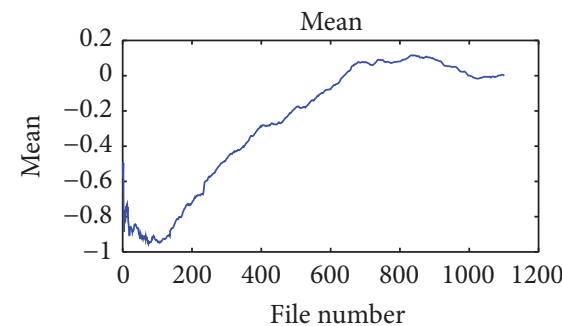

(a)

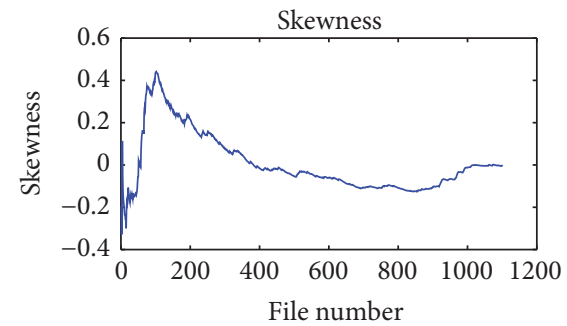

(d)

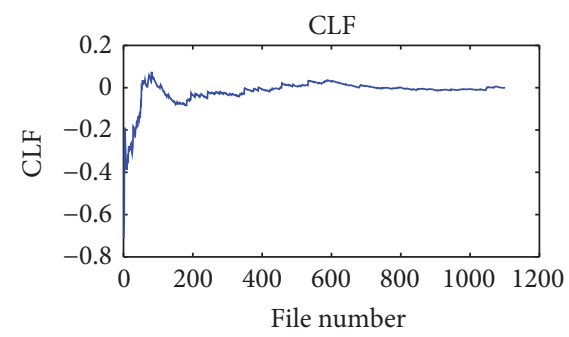

(g)

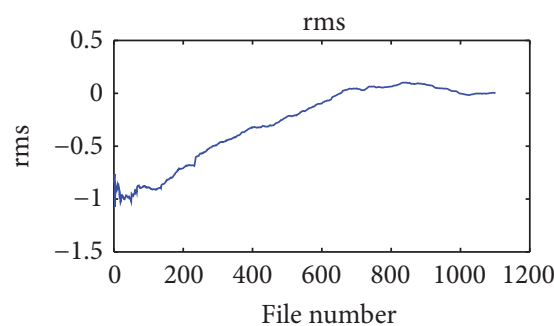

(b)

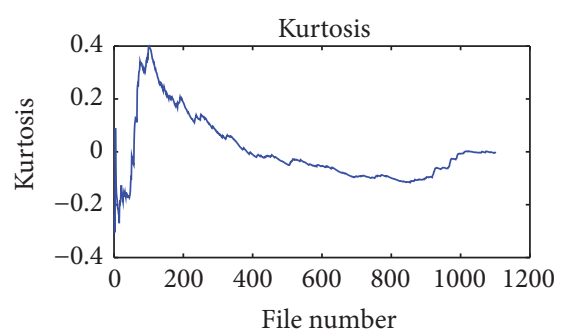

(e)

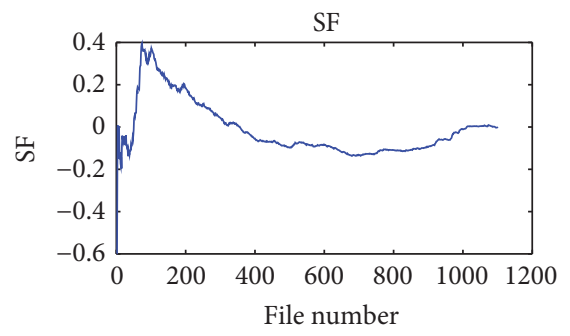

(h)

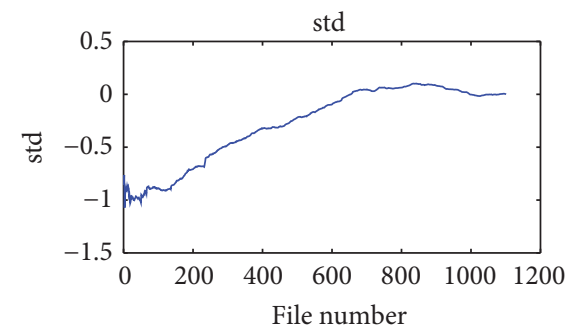

(c)

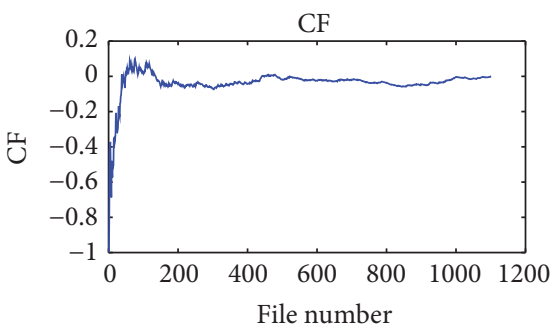

(f)

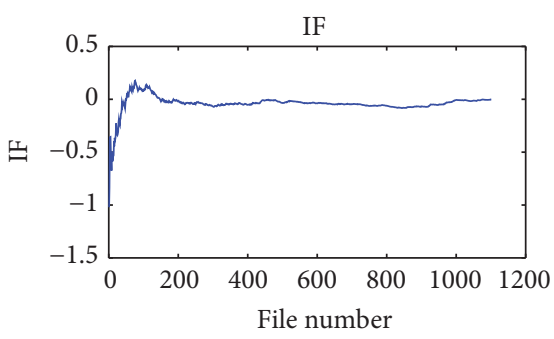

(i)

Figure 7: The nine statistical feature indicators from Figure 6 after normalization, processed with the moving average (mean, rms, std, skewness, kurtosis, CF, CLF, SF, and IF from (a)-(i)).

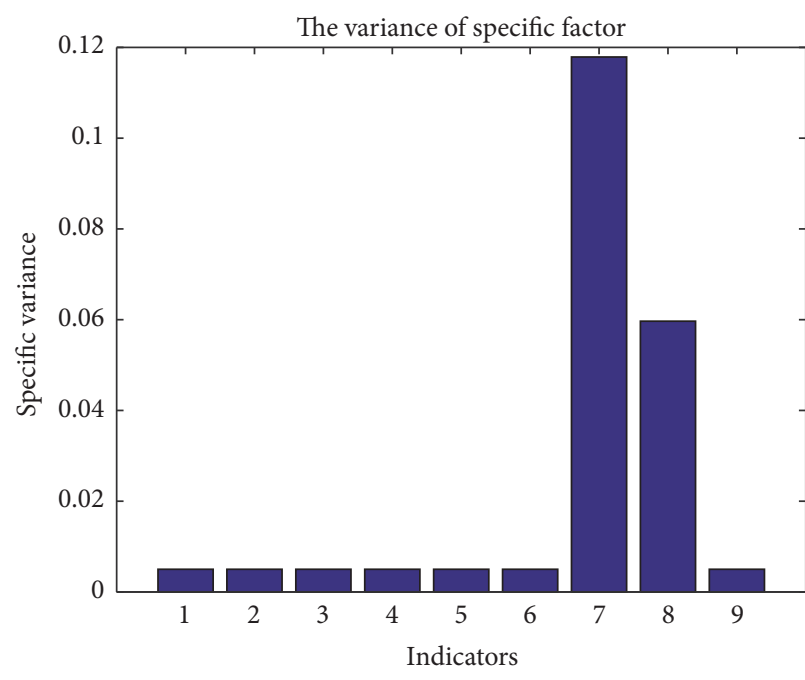

(a)

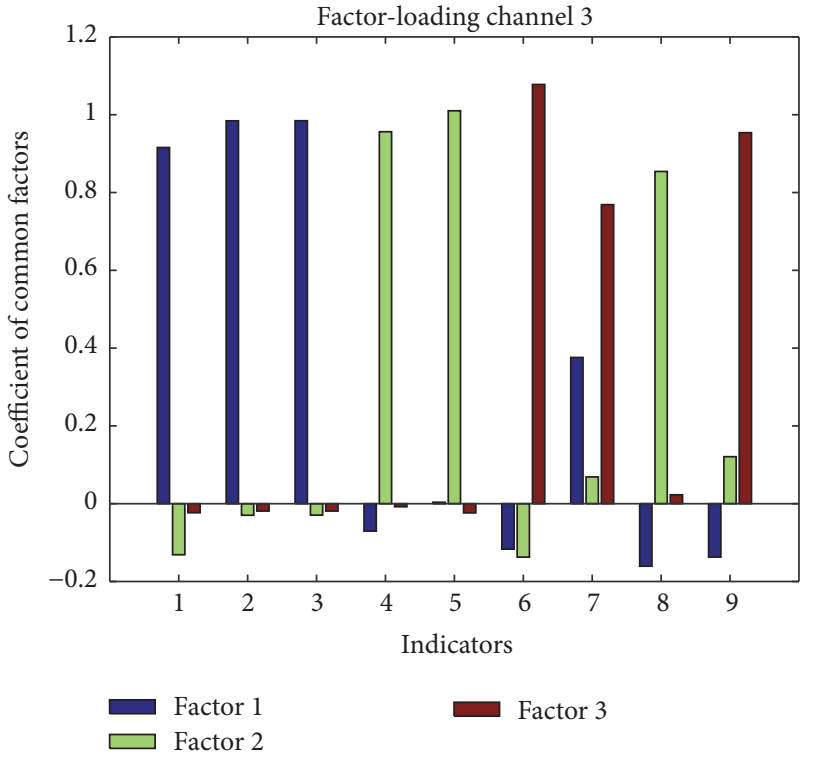

(b)

Figure 8: (a) The specific variances of indicators. (b) The coefficients of the common factors. 


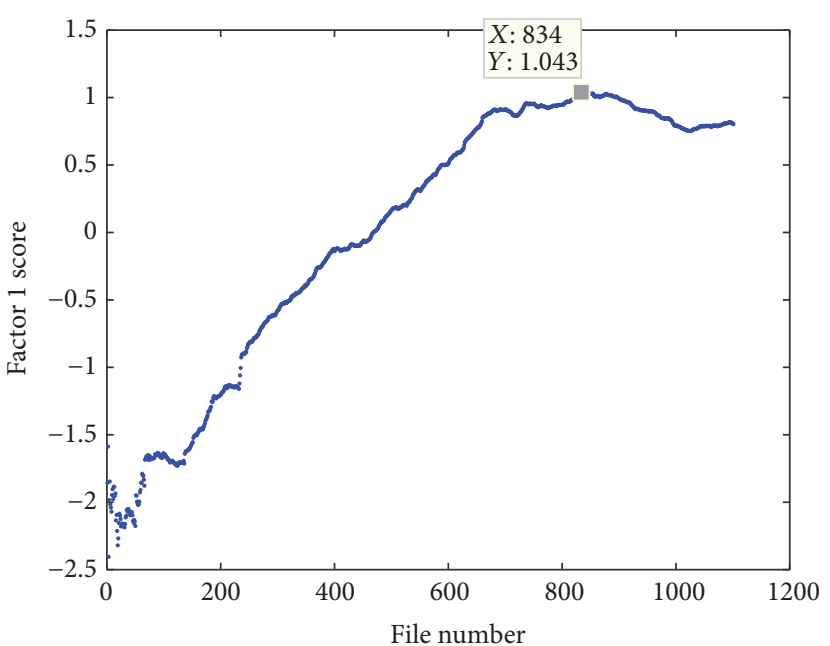

- Factor 1

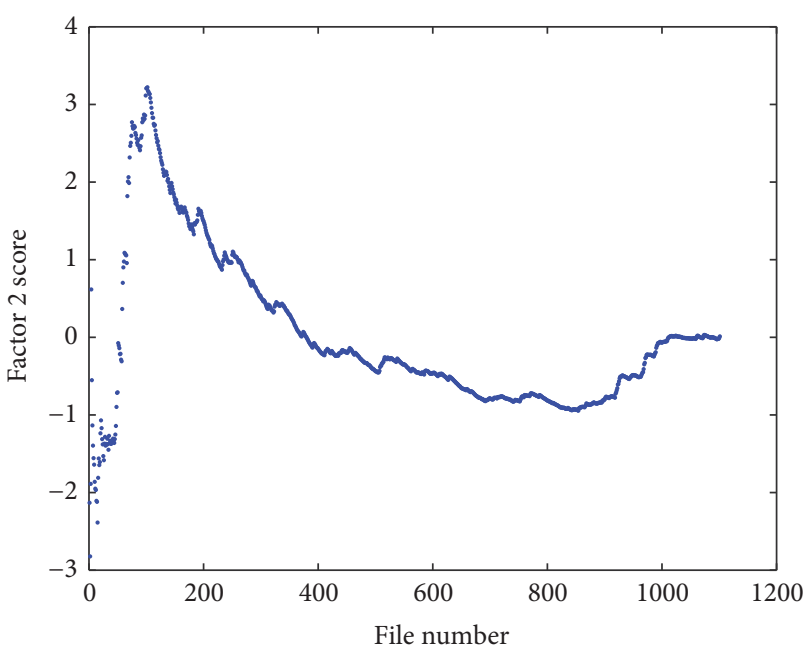

- Factor 2

(a)

(b)

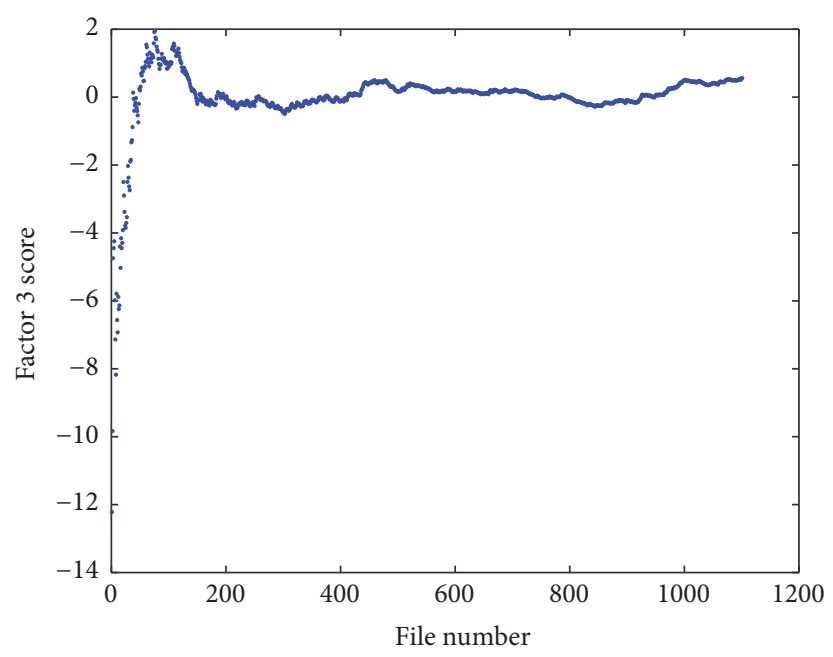

- Factor 3

(c)

FIGURE 9: The results of the EFABII in frequency domain for (a) factor 1, (b) factor 2, and (c) factor 3, respectively.

TABLE 1: Parameters of factors results.

\begin{tabular}{lcccccc}
\hline \multirow{2}{*}{ Type } & \multicolumn{2}{c}{ Time domain } & \multicolumn{3}{c}{ Frequency domain } \\
& Real value & Factor 2 & Principle component 1 & Real value & Factor 1 & Principle component 1 \\
\hline Standard deviation & 0.23 & 0.65 & 0.32 & 0.23 & 0.64 & 0.42 \\
Slope & 0.0013 & 0.0037 & 0.0018 & 0.0013 & 0.0036 & 0.0024 \\
P-P (peak-peak) & 0.77 & 2.16 & 1.12 & 0.77 & 2.14 & 1.44 \\
\hline
\end{tabular}

indicator to describe the slurry pumps' wear degradation propagation performance.

In summary, factor 1 in the frequency domain is chosen as the new EFABII for assessing the slurry pumps' performance degradation and damage growth trends.

4.3. Comparison between Enhanced Factor Analysis and Principle Component Analysis. The comparison between the selected factors, the principle components, and real values in both time and frequency domains are shown in Figures 10 and 11, and Table 1 . Three factors in each domain are, respectively, obtained by using the EFA method, described in Sections 4.1 and 4.2. However, there must be some global criteria to determine the factor that will be the best EFABII and which fulfills the requirements for assessing slurry pump performance degradation. Specific numerical parameters are required to assess the effectiveness and efficiency of the selected factors' compliance with wear degradation performance. 


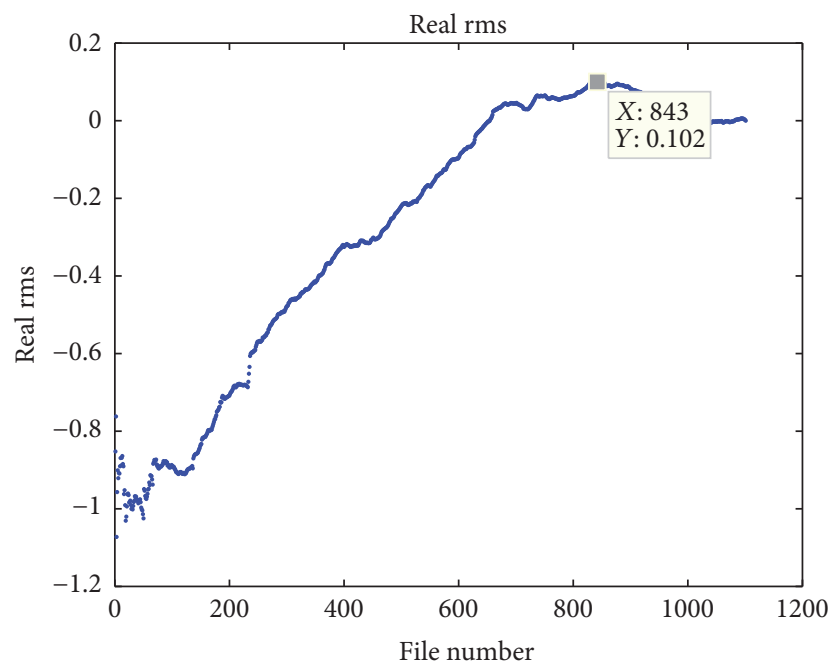

(a) Real rms

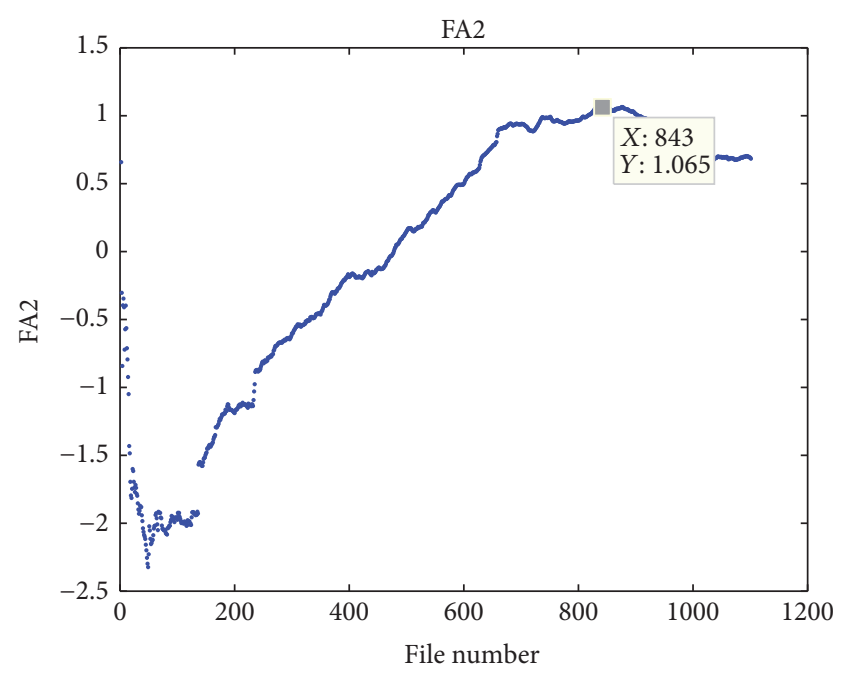

(b) Factor 2

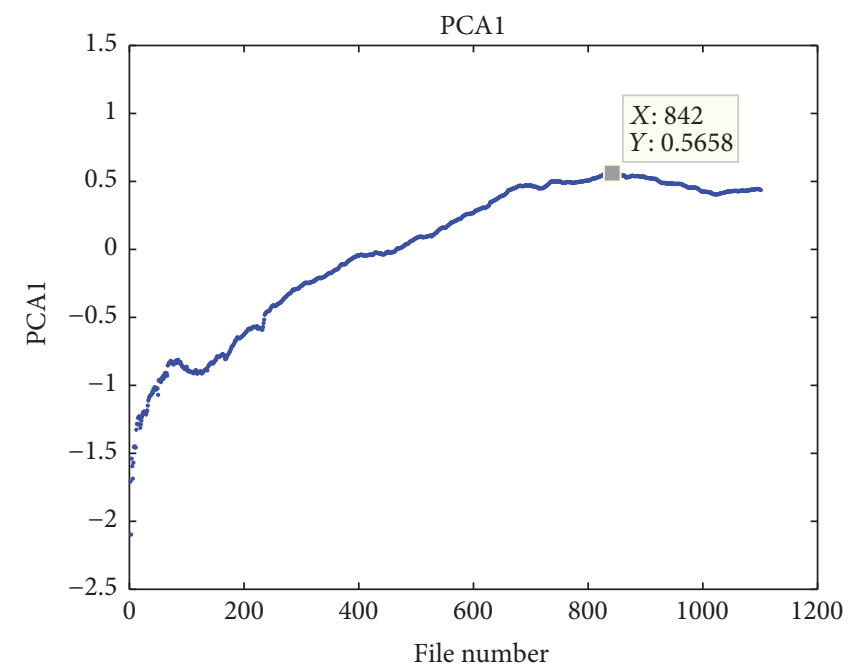

(c) Principle component 1

FIGURE 10: Comparison among the selected factor, principle component, and real values in time domain.

To investigate the criteria for selecting the optimum EFABII, the mean, std, and the slope of the linear regressions of the various factors are required, as shown in Table 1. It is found that by self-defining these three indexes, the most representable factor can be automatically selected. Through the analysis presented above, factor 2 in the time domain and factor 1 in the frequency domain are selected as the EFABII, as they have similar trends to each of the original impeller indicators. This criterion can be further studied and modified.

\section{Conclusions}

A typical degradation trend can be divided by three stages. In the beginning of the pump life cycle, the impeller is new and smooth, hence generating little vibration. Then, during the continuous operation, due to bombarding with slurries, the impeller's surface becomes rougher in time, which results in continuous growing of vibration. The amplitude of vibration will grow to a peak and then gradually drop in the third life stage. It is because after severe wear of the impeller, the vane length becomes shorter which results in free flow of slurries to the outlet of the pump, causing less vibration and pressure drop. At this stage, although the slurry is still operating, but the pressure head or pump efficiency will drop substantially. That is, after reaching the peak of the degradation trend as shown in the bottom diagram, the impellers should be replaced to maintain the expected slurry pump operation and efficiency again. In summary, the collected vibration data and our developed methods can help to draw the impeller's degradation trend due to wear for a particular slurry pump under a certain operating condition. With such degradation trend and its peak appearing, one can start to use other methods to estimate the remaining useful live of each unique slurry pump.

In this paper, a combined low-pass moving averaging filter and an enhanced factor analysis method are presented and applied to analyze the wear degradation trend of slurry 


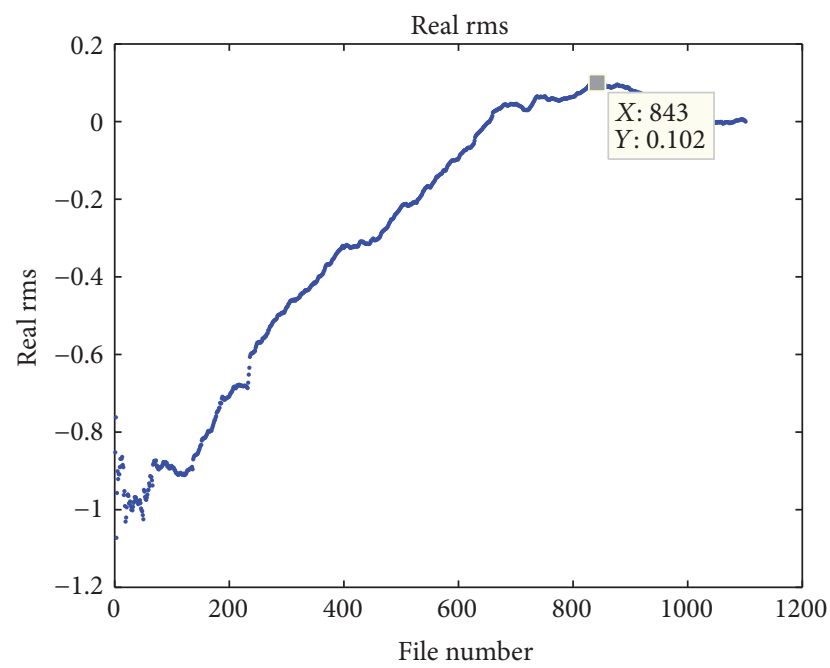

(a) Real rms

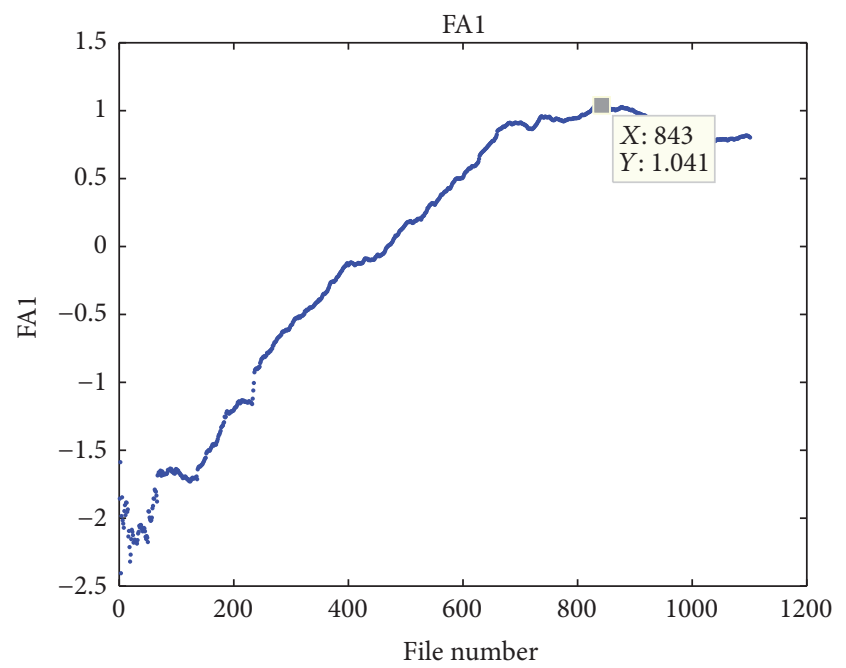

(b) Factor 1

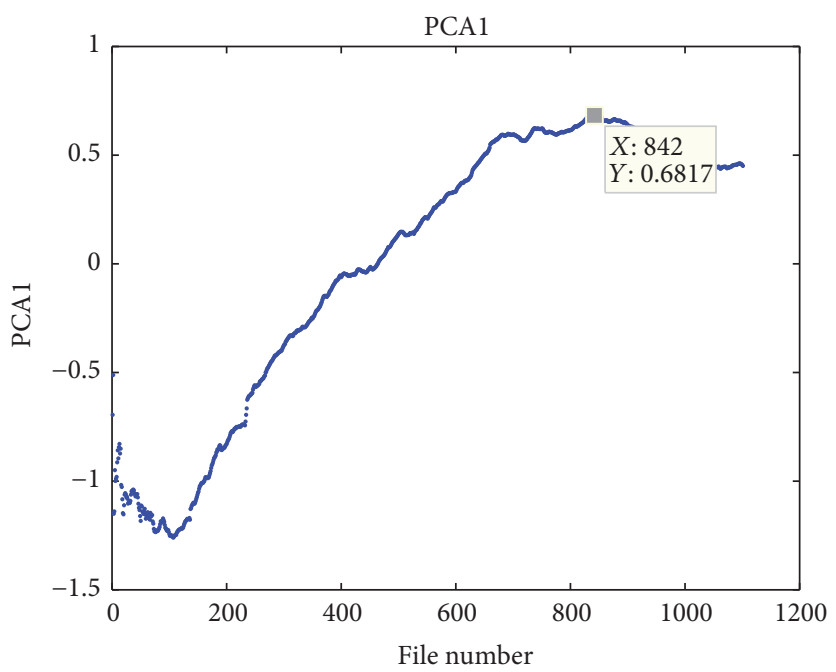

(c) Principle component 1

FIGURE 11: Comparison among the selected factor, principle component, and real values in frequency domain.

pumps. The nine initial time domain features were replaced by a single feature, factor 2 , and the nine frequency domain features were replaced by factor 1 . The closer the absolute coefficients of the common factors to " 1 ," the larger the correlation will be with that kind of feature. Therefore, the method proposed in this study can be used to automatically select the most effective new indicators for monitoring the slurry pumps' wear degradation propagation performance and to decrease the dimensionality of multivariate data. In other words, these two automatically selected factors can be used to monitor the trends of vibration signal conditions in temporal and spectral domains, instead of the 18 features extracted from the processed data. The enhanced factor analysis based impeller indicator (EFABII) had been validated by experimental studies as an effective indicator for the performance degradation assessments of slurry pumps. A new factor selection criterion combining the mean, the standard deviation, and the slope throughout the life stage of pumps is also presented.

\section{Competing Interests}

The authors declare that there are no competing interests regarding the publication of this paper.

\section{Acknowledgments}

The work described in this paper was fully supported by a grant from the Research Grants Council (Project no. 11201315) and a grant from the Innovation and Technology Commission (ITC) (Project no. ITS/061/14FP) of the Government of the Hong Kong Special Administrative Region (HKSAR), China.

\section{References}

[1] J. Qu and M. J. Zuo, "Support vector machine based data processing algorithm for wear degree classification of slurry pump systems," Measurement, vol. 43, no. 6, pp. 781-791, 2010. 
[2] X. Zhao, Q. Hu, Y. Lei, and M. J. Zuo, "Vibration-based fault diagnosis of slurry pump impellers using neighbourhood rough set models," Proceedings of the Institution of Mechanical Engineers Part C: Journal of Mechanical Engineering Science, vol. 224, no. 4, pp. 995-1006, 2010.

[3] D. Wang and P. W. Tse, "Prognostics of slurry pumps based on a moving-average wear degradation index and a general sequential Monte Carlo method," Mechanical Systems and Signal Processing, vol. 56, pp. 213-229, 2015.

[4] X. Zhao, M. J. Zuo, and T. H. Patel, "Generating an indicator for pump impeller damage using half and full spectra, fuzzy preference-based rough sets and PCA," Measurement Science and Technology, vol. 23, no. 4, Article ID 045607, 2012.

[5] Q. Miao, D. Wang, and M. Pecht, "A probabilistic description scheme for rotating machinery health evaluation," Journal of Mechanical Science and Technology, vol. 24, no. 12, pp. 24212430, 2010.

[6] D. Wang, Q. Miao, and R. Kang, "Robust health evaluation of gearbox subject to tooth failure with wavelet decomposition," Journal of Sound and Vibration, vol. 324, no. 3-5, pp. 1141-1157, 2009.

[7] D. Wang, P. W. Tse, W. Guo, and Q. Miao, "Support vector data description for fusion of multiple health indicators for enhancing gearbox fault diagnosis and prognosis," Measurement Science and Technology, vol. 22, no. 2, Article ID 025102, 2011.

[8] Q. Miao, C. Tang, W. Liang, and M. Pecht, "Health assessment of cooling fan bearings using wavelet-based filtering," Sensors, vol. 13, no. 1, pp. 274-291, 2013.

[9] Y. Lei, D. Kong, J. Lin, and M. J. Zuo, "Fault detection of planetary gearboxes using new diagnostic parameters," Measurement Science and Technology, vol. 23, no. 5, Article ID 055605, 2012.

[10] P. W. Tse and D. Wang, "Enhancing the abilities in assessing slurry pumps' performance degradation and estimating their remaining useful lives by using captured vibration signals," Journal of Vibration and Control, 2015.

[11] P. W. Tse and D. Wang, "Extraction of principal components from multiple statistical features for slurry pump performance degradation assessment," Lecture Notes in Control and Information Sciences, vol. 20, pp. 131-141, 2015.

[12] Y.-W. Chang, Y.-C. Wang, T. Liu, and Z.-J. Wang, "Fault diagnosis of a mine hoist using PCA and SVM techniques," Journal of China University of Mining and Technology, vol. 18, no. 3, pp. 327-331, 2008.

[13] J. Harmouche, C. Delpha, and D. Diallo, "Incipient fault detection and diagnosis based on Kullback-Leibler divergence using principal component analysis: Part II," Signal Processing, vol. 109, pp. 334-344, 2015.

[14] J. Harmouche, C. Delpha, and D. Diallo, "Incipient fault detection and diagnosis based on Kullback-Leibler divergence using Principal Component Analysis: Part i," Signal Processing, vol. 94, no. 1, pp. 278-287, 2014.

[15] J.-M. Lee, S. J. Qin, and I.-B. Lee, "Fault detection and diagnosis based on modified independent component analysis," AIChE Journal, vol. 52, no. 10, pp. 3501-3514, 2006.

[16] J. F. Hair, W. C. Black, B. J. Babin, R. E. Anderson, and R. L. Tatham, Multivariate Data Analysis, vol. 6, Pearson Prentice Hall, Upper Saddle River, NJ, USA, 2006.

[17] G. I. Butnaru, "The method of ethnographic and content analysis in determining development factors of economic and managerial tourism performance," Procedia Economics and Finance, vol. 20, pp. 104-111, 2015.
[18] C. Safdari, N. J. Scannell, and R. Ohanian, "A statistical approach to peer-groupings; the case of banks in Armenia," The Journal of American Academy of Business, Cambridge, vol. 6, pp. 24-31, 2005.

[19] R. Schulze, A.-L. Hilger, and P. M. Engelberg, "Factor analysis and latent variable models in personality psychology," in International Encyclopedia of the Social \& Behavioral Sciences, J. D. Wright, Ed., pp. 703-708, Elsevier, Oxford, UK, 2nd edition, 2015.

[20] B. K. Miller, D. L. Smart, and P. L. Rechner, "Confirmatory factor analysis of the machiavellian personality scale," Personality and Individual Differences, vol. 82, pp. 120-124, 2015.

[21] R. O. Mueller and G. R. Hancock, "Factor analysis and latent structure analysis: confirmatory factor analysis," in International Encyclopedia of the Social \& Behavioral Sciences (Second Edition), J. D. Wright, Ed., pp. 686-690, Elsevier, Oxford, UK, 2015.

[22] M. Nokhandan, G. A. F. Ghalhar, and M. Mousavi-Ba, "The Application of Factor Analysis and Artificial Neural Networks in Predicting Spring Precipitation by Means of Climatic Parameters of the Upper Levels of Atmosphere," Trends in Applied Sciences Research, vol. 4, no. 2, pp. 85-97, 2009.

[23] J. Chaijaruwanich, J. Khamphachua, S. Prasitwattanaseree, S. Warit, and P. Palittapongarnpim, "Application of factor analysis on Mycobacterium tuberculosis transcriptional responses for drug clustering, drug target, and pathway detections," in Advanced Data Mining and Applications, vol. 4093 of Lecture Notes in Computer Science, pp. 835-844, Springer, Berlin, Germany, 2006.

[24] D. W. Apley and J. Shi, "A factor-analysis method for diagnosing variability in multivariate manufacturing processes," Technometrics, vol. 43, no. 1, pp. 84-95, 2001.

[25] Y. Zhang, Z. Zhao, Y. Sun, and Z. Wang, "Fault factor analysis in complicated electrical engineering," Electronics \& Electrical Engineering, vol. 19, no. 10, pp. 17-20, 2013.

[26] L. S. Kuravsky, P. A. Marmalyuk, S. N. Baranov, and N. I. Baranov, "Wavelet-based confirmatory factor analysis: monitoring of damage accumulation factors," Applied Mathematical Sciences, vol. 9, no. 25-28, pp. 1245-1263, 2015.

[27] A. C. Rencher, Methods of multivariate analysis, Wiley Series in Probability and Statistics, Wiley-Interscience, Second edition, 2002.

[28] D. Child, The Essentials of Factor Analysis, Cassell Educational, London, UK, 1990.

[29] R. A. Johnson and D. W. Wichern, Applied Multivariate Statistical Analysis, vol. 4, Prentice Hall, Englewood Cliffs, NJ, USA, 1992.

[30] J. Yu, "Bearing performance degradation assessment using locality preserving projections and Gaussian mixture models," Mechanical Systems and Signal Processing, vol. 25, no. 7, pp. 2573-2588, 2011.

[31] H. Qiu, J. Lee, J. Lin, and G. Yu, "Robust performance degradation assessment methods for enhanced rolling element bearing prognostics," Advanced Engineering Informatics, vol. 17, no. 3-4, pp. 127-140, 2003.

[32] Z. Shen, Z. He, X. Chen, C. Sun, and Z. Liu, "A monotonic degradation assessment index of rolling bearings using fuzzy support vector data description and running time," Sensors, vol. 12, no. 8, pp. 10109-10135, 2012. 


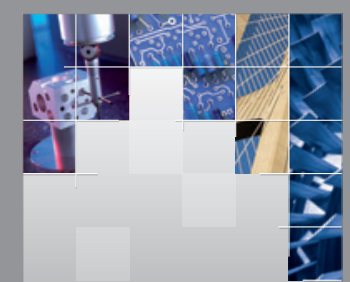

\section{Enfincering}
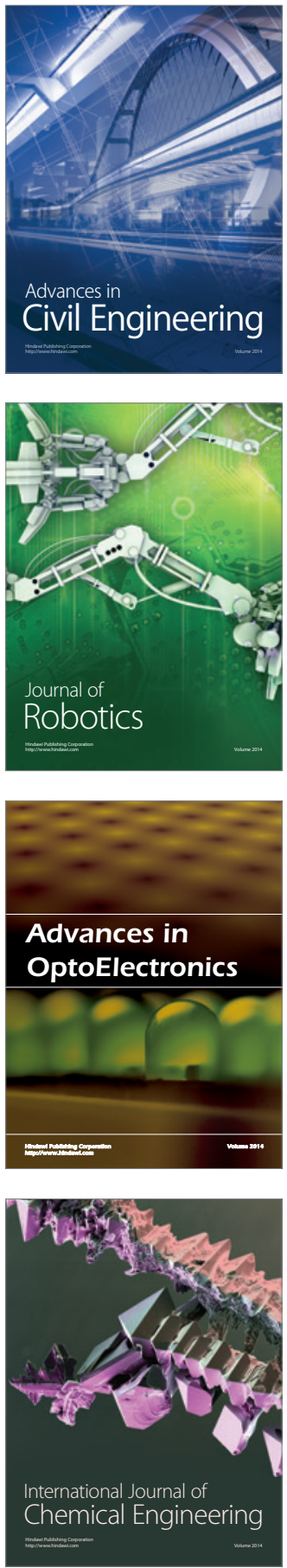

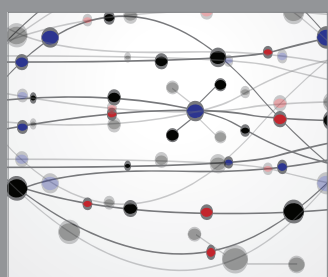

The Scientific World Journal

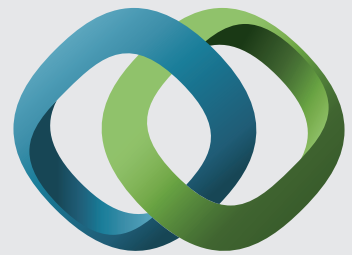

\section{Hindawi}

Submit your manuscripts at

https://www.hindawi.com
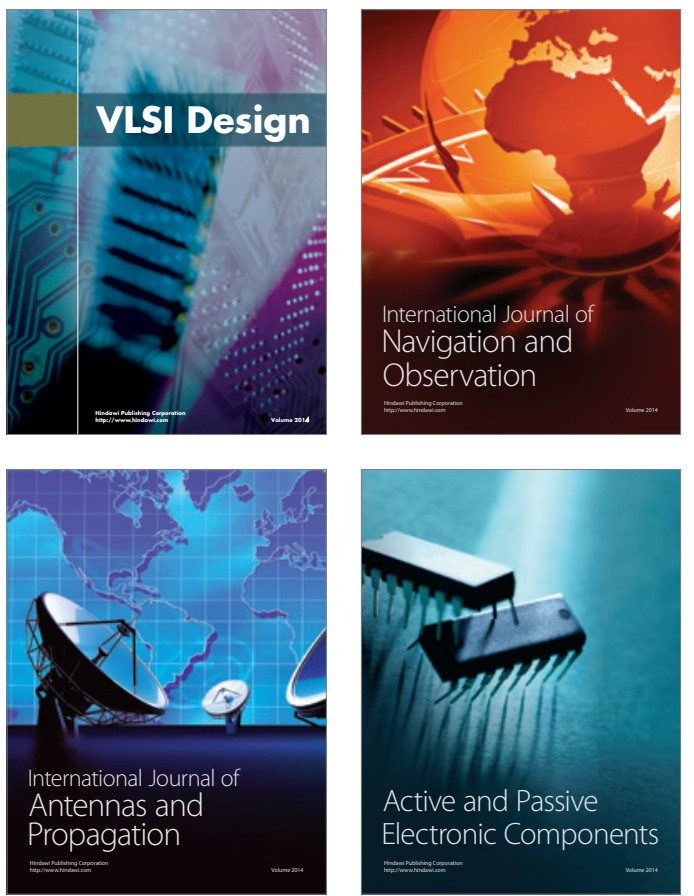
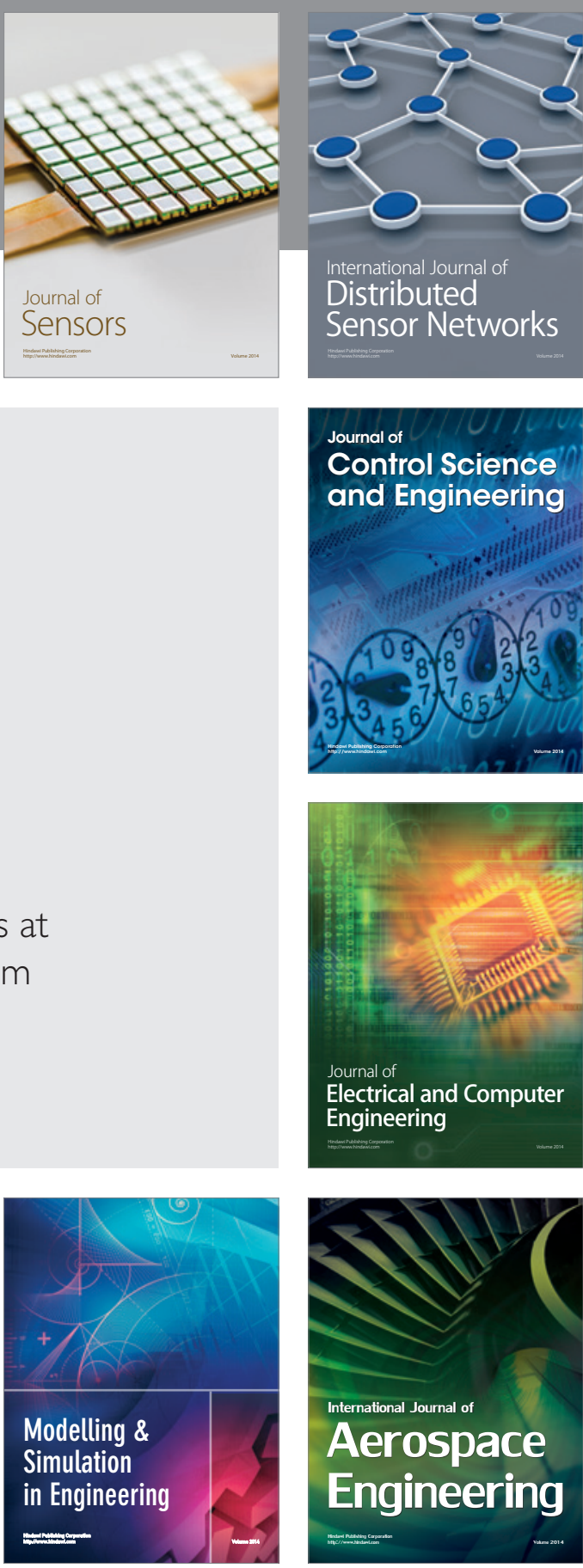

International Journal of

Distributed

Sensor Networks

$-$

Joumal of

Control Science

and Engineering
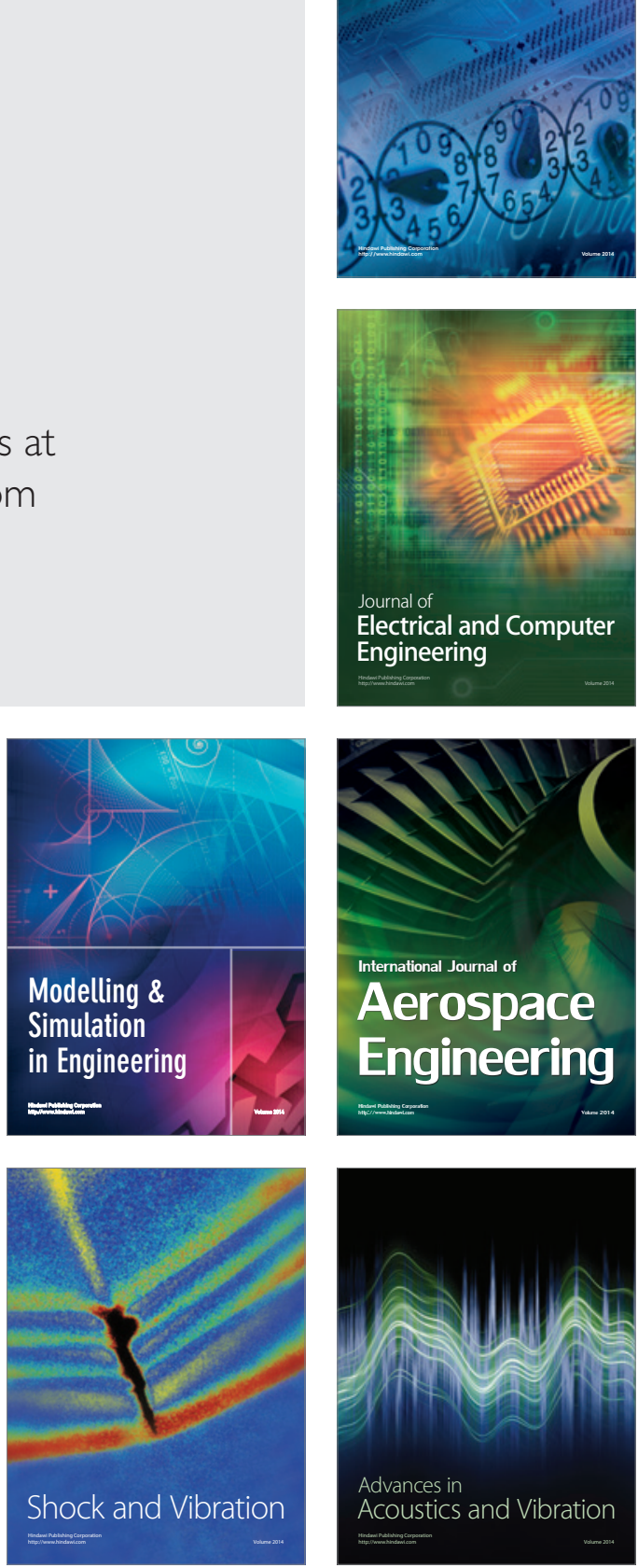